\title{
Relationship between Gleason score and apparent diffusion coefficients of diffusion-weighted magnetic resonance imaging in prostate cancer patients
}

\author{
Tae Heon Kim, MD; ${ }^{1}$ Chan Kyo Kim, MD; ${ }^{2}$ Byung Kwan Park, MD; Hwang Gyun Jeon, MD, ${ }^{3}$ \\ Byung Chang Jeong, MD, ${ }^{3}$ Seong II Seo, MD,; Hyun Moo Lee, MD, Han Yong Choi, MD,; Seong Soo Jeon, MD
}

'Department of Urology, Samsung Changwon Hospital, Sungkyunkwan University School of Medicine, Changwon, Korea; ${ }^{2}$ Department of Radiology, Samsung Medical Centre, Sungkyunkwan University School of Medicine, Seoul, Korea; ${ }^{3}$ Department of Urology, Samsung Medical Centre, Sungkyunkwan University School of Medicine, Seoul, Korea

Cite as: Can Urol Assoc J 2016;10(11-12):E377-82. http://dx.doi.org/10.5489/cuaj.3896 Published online November 10, 2016.

\section{Abstract}

Introduction: We assessed the correlation between the apparent diffusion coefficient (ADC) and pathological Gleason score (GS) of prostate cancer patients.

Methods: A total of 125 patients who underwent multiparametric magnetic resonance imaging before radical prostatectomy for prostate cancer were included in this study. ADC values were compared with different GS. We used receiver operating characteristic analysis and determined the ADC cutoff value to differentiate tumours with a GS of 6 from those with a GS $\geq 7$.

Results: We identified 34 patients $(27.2 \%)$ with a GS of $6 ; 33$ patients $(26.4 \%)$ with a GS of $7 ; 22$ patients $(17.6 \%)$ with a GS of 8 ; and 36 patients $(28.8 \%$ ) with a GS of $\geq 9$. The mean ADC value for disease with a GS of 6 was $0.914 \pm 0.161 \times 10^{-3} \mathrm{~mm}^{2} / \mathrm{s}$; GS of 7: $0.741 \pm 0.164 \times 10^{-3} \mathrm{~mm}^{2} / \mathrm{s}$; GS of $8: 0.679 \pm 0.130 \times 10^{-3}$ $\mathrm{mm}^{2} / \mathrm{s}$; and GS of $\geq 9$ : $0.593 \pm 0.089 \times 10^{-3} \mathrm{~mm}^{2} / \mathrm{s}$. An ADC value of $0.830 \times 10^{-3} \mathrm{~mm}^{2} / \mathrm{s}$ was the best cutoff value to identify prostate cancer with a GS of 6 .

Conclusions: We observed an inverse relationship between GS and ADC value. Moreover, a cutoff ADC value may help differentiate disease with a GS of 6 from disease with a GS $\geq 7$.

\section{Introduction}

Prostate cancer is one of the most common cancers among men and the second most common cause of death by cancer in industrialized countries. ${ }^{1}$ Owing to the biologic heterogeneity of prostate cancer, which ranges from indolent to highly aggressive, accurate classification of aggressiveness is essential for selecting an appropriate course of treatment. The optimal strategy of prostate cancer management is to identify and treat patients with aggressive disease who would benefit from radical therapy, but to avoid overtreatment in patients for whom prostate cancer is unlikely to be lifethreatening. Although considerable efforts have been made to characterize the aggressiveness of prostate cancer, ${ }^{2-5}$ there are currently no reliable, non-invasive tests to accurately assess prostate cancer aggressiveness.

Pathological Gleason score (GS) is a useful parameter for predicting prostate cancer aggressiveness, with a higher GS indicating increased biological aggressiveness.6,7 However, determination of GS is based on transrectal ultrasoundguided prostate biopsy, which is associated with sampling error and severe adverse events; ${ }^{8-10}$ in particular, upgrading a patient with a higher GS from biopsy to prostatectomy specimen occurs in up to one-third of patients. ${ }^{11,12}$

Recently, multiparametric magnetic resonance imaging (mpMRI), including diffusion-weighted imaging (DWI), has emerged as an important modality in prostate cancer diagnosis. ${ }^{13-15}$ Several studies have identified the role of the apparent diffusion coefficient (ADC) value, obtained from DWI sequences, as a novel tool that may improve the diagnosis and management of prostatic cancer. These studies focused on the association between ADC value and GS. ${ }^{16-20} \mathrm{ADC}$ is a relatively simple metric that can be calculated with standard 1.5- and 3.0-Tesla (T) mpMRI platforms. Furthermore, ADC provides quantitative information on tumour characteristics that indirectly predict tumour aggressiveness. ${ }^{21}$

To gain better insight and evidence to support the use of ADC value in predicting prostate cancer aggressiveness, we evaluated the association between prostate cancer GS and ADC values obtained from diffusion-weighted MR imaging of the prostate. We sought to determine which ADC metric best differentiates GS 6 prostate cancer lesions from prostate cancer lesions with a GS of 7 or higher. 
Kim et al.

\section{Methods}

\section{Study population}

This retrospective study was approved by our institutional review board, which also waived the requirement for informed consent. Inclusion criteria were as follows: patients with a single prostate cancer lesion and with a cancer volume of more than $0.5 \mathrm{~cm}^{3}$ according to the radical prostatectomy specimen, and patients who underwent preoperative mpMRI for staging. Radical prostatectomy was performed by a single surgeon (S.S.J.) at our institution. MRI examination was performed at least three weeks after prostate biopsy. Patients who had undergone prior hormonal or radiation therapy were excluded. Patients who had incomplete medical records or patients for whom MRI was performed at an outside institution were also excluded.

\section{MRI protocol and imaging analysis}

All patients were examined using a 3.0-T MRI system (Intera Achieva 3T, Philips Medical System, Best, Netherlands) equipped with a six-channel, phased-array coil. The MRI protocol was composed of DW-MRI and dynamic contrast-enhanced imaging, in addition to a routine prostatic MRI (T2-weighted and T1-weighted imaging). DW-MRI was performed with b-values of 0 and $1000 \mathrm{~s} / \mathrm{mm}^{2}$ along three orthogonal directions of motion-probing gradients. Automated calculation of ACD maps was performed using the manufacturer's software, which was then used to obtain a monoexponential fit of the data.

All MR images were archived using a picture archiving and communication system (PACS; PathSpeed Workstation; GE Medical Systems, Milwaukee, WI, U.S.). ADC values were measured by a single experienced urologist who was aware of all clinical and histopathological findings, including tumour location. ADC values were measured by regions of interest (ROIs) drawn on possible cancer areas based on MRI findings previously interpreted by two experienced genitourinary radiologists. ADC values were measured twice at the same site and the results were averaged. A prostate cancer was defined on each MRI as follows: an abnormally low signal intensity region relative to the adjacent parenchyma on T2-weighted imaging; a high signal intensity region at $b$ values of $1000 \mathrm{~s} / \mathrm{mm}^{2}$ of DWI with a low ADC value on the ADC map images; and a region with early wash-in and wash-out of contrast material relative to the adjacent parenchyma on dynamic contrast enhanced MRI. T1-weighted images were available for interpreting post-biopsy hemorrhage (an area of high signal intensity on T1-weighted imaging). Circular ROls were manually generated using the ADC maps for prostate cancer lesions for each patient. The median ROI area in the study was $33.6 \mathrm{~mm}^{2}$ (interquartile range [IQR], $27.0-40.9 \mathrm{~mm}^{2}$ ).

\section{Pathology analysis}

All prostatectomy specimens were marked with India ink and fixed overnight in $10 \%$ buffered formalin. Transverse step sections were obtained at 3-4 mm intervals in a plane perpendicular to the prostatic urethra. Routine pathologic examination for all specimens was performed by an experienced genitourinary pathologist who was unaware of the MRI findings. Pathological outcomes were recorded with a standard reporting protocol. Pathological tumour volumes were calculated by the ellipsoid estimation method suggested by Noguchi et al ${ }^{22}$ as follows: tumour volume $\left(\mathrm{cm}^{3}\right)$ $=\varpi / 6 \times$ width $\times$ height $\times$ length .

\section{Statistical analysis}

Descriptive data are presented as frequencies, while categorical variables are presented as percentages. The Shapiro-Wilk normality test was used to investigate the normal distribution of continuous variables. Continuous and non-normally distributed variables are presented as medians with IQRs. Student's t-test or the Mann-Whitney $U$ test was used to compare differences in ADC values in pairs according to GS. We used the receiver operating characteristic (ROC) curve to evaluate the diagnostic performance of ADC values in the differentiation of prostate cancer with a GS of 6 from prostate cancer with a GS $\geq 7$, as well as to determine the ADC cutoff level that provided the highest diagnostic performance.

Diagnostic performance was assessed by calculating the area under the curve $(A \cup C)$. We also performed internal validation with bootstrapping in order to estimate the optimism in our model. In 1000 bootstrap resamples, the concordance index of the model was estimated and tested in the original sample. All reported $p$ values in this study were two-sided and $p$ values $<0.05$ were considered statistically significant. Statistical analyses were performed with SPSS ${ }^{\circledR}$ for Windows, version 21.0 (IBM Corporation, NY, U.S).

\section{Results}

A total of 125 patients were enrolled in this study. Table 1 shows the characteristics of the enrolled study population. The median patient age was 66.0 year (IQR 60.0-70.0) and the median prostate-specific antigen (PSA) was 7.1 (IQR 4.5-12.4). The mean tumour volume was $2.96 \mathrm{~cm}^{3}$ (IQR 0.87-10.00). With respect to histopathological analysis, we identified 34 patients with a GS of 6; 33 patients with a GS of $7 ; 22$ patients with a GS of $8 ; 23$ patients with a GS of 9 ; and 13 patients with a GS of 10 . The mean ADC value was 0.914 


\begin{tabular}{lc}
\hline \multicolumn{2}{l}{ Table 1. Characteristics of study population } \\
\hline Characteristics & Value \\
\hline Age, years (IQR) & $66.0(60.0-70.0)$ \\
Prostate-specific antigen, $\mathrm{ng} / \mathrm{ml}(\mathrm{IQR})$ & $7.12(4.50-12.42)$ \\
Tumour volume, $\mathrm{cm}^{3}$ (IQR) & $2.96(0.87-10.00)$ \\
Pathologic stage, $\mathrm{n}(\%)$ & \\
T2a & $19(15.2)$ \\
T2b & $1(0.8)$ \\
T2c & $39(31.2)$ \\
T3a & $36(28.8)$ \\
T3b & $25(20.0)$ \\
T4 & $5(4.0)$ \\
Gleason score, $\mathrm{n}(\%)$ & \\
6 & $34(27.2)$ \\
7 & $33(26.4)$ \\
8 & $22(17.6)$ \\
9 & $23(18.4)$ \\
10 & $13(10.4)$ \\
\hline
\end{tabular}

Data are presented as median (interquartile range [IQR]) or $\mathrm{n}(\%)$.

$\pm 0.161 \times 10^{-3} \mathrm{~mm}^{2} / \mathrm{s}$ for a GS of $6 ; 0.741 \pm 0.164 \times 10^{-3} \mathrm{~mm}^{2} / \mathrm{s}$ for a GS of 7; $0.679 \pm 0.130 \times 10^{-3} \mathrm{~mm}^{2} / \mathrm{s}$ for a GS of 8 ; and $0.593 \pm 0.089 \times 10^{-3} \mathrm{~mm}^{2} / \mathrm{s}$ for $\mathrm{GS} \geq 9$. Representative cases with a GS of $6(3+3)$ prostate cancer and a GS of $8(4+4)$ prostate cancer are shown in Figs. 1 and 2. The differences between the ADC values obtained for all GS groups $(6,7$, 8 , and $\geq 9$ ) were analyzed in pairs and there were significant differences between all of the GS groups with the exception of GS 7 and GS 8 (Fig. 3). Furthermore, the mean ADC value of tumours with a GS of 7 or higher $\left(0.668 \times 10^{-3} \mathrm{~mm}^{2} / \mathrm{s}\right)$ was significantly lower than that of tumours with a GS of 6 $\left(0.914 \times 10^{-3} \mathrm{~mm}^{2} / \mathrm{s}\right)(\mathrm{p}<0.001)$ (Fig. 4).

Fig. 5 shows the ROC curve for the cutoff ADC assessment, the AUC of which was 0.880 (95\% confidence interval $0.813-0.946)$. In addition, an ADC of $0.830 \times 10^{-3} \mathrm{~mm}^{2} / \mathrm{s}$ was the best cutoff value to identify prostate cancer with a GS of 6 , which had a sensitivity and specificity of $79.4 \%$ and $87.5 \%$, respectively. Through bootstrap validation, the concordance indexes of the model in predicting a GS of 6 were 0.826 and 0.832 in the training and testing data sets, respectively.

\section{Discussion}

$m p M R I$ is rapidly gaining importance for detecting and characterizing prostate cancer. In particular, DWI and ADC values are regarded as reliable non-invasive tools for assessing tumour aggressiveness in prostate cancer. Our study demonstrated that ADC values were inversely correlated with GS in prostate cancer and that it was feasible to discriminate among pathologic GS 6 disease according to ADC values. This finding was consistent with earlier studies that reported that ADC values may be useful in differentiating patients with high- or intermediate-risk prostate cancer from those with a low risk prostate cancer. ${ }^{16,23-26}$ In addition, several previous studies have demonstrated that high GS disease has a lower ADC value than that of low GS disease. Specifically, Nagarajan et $\mathrm{al}^{17}$ compared ADC values with three groups of

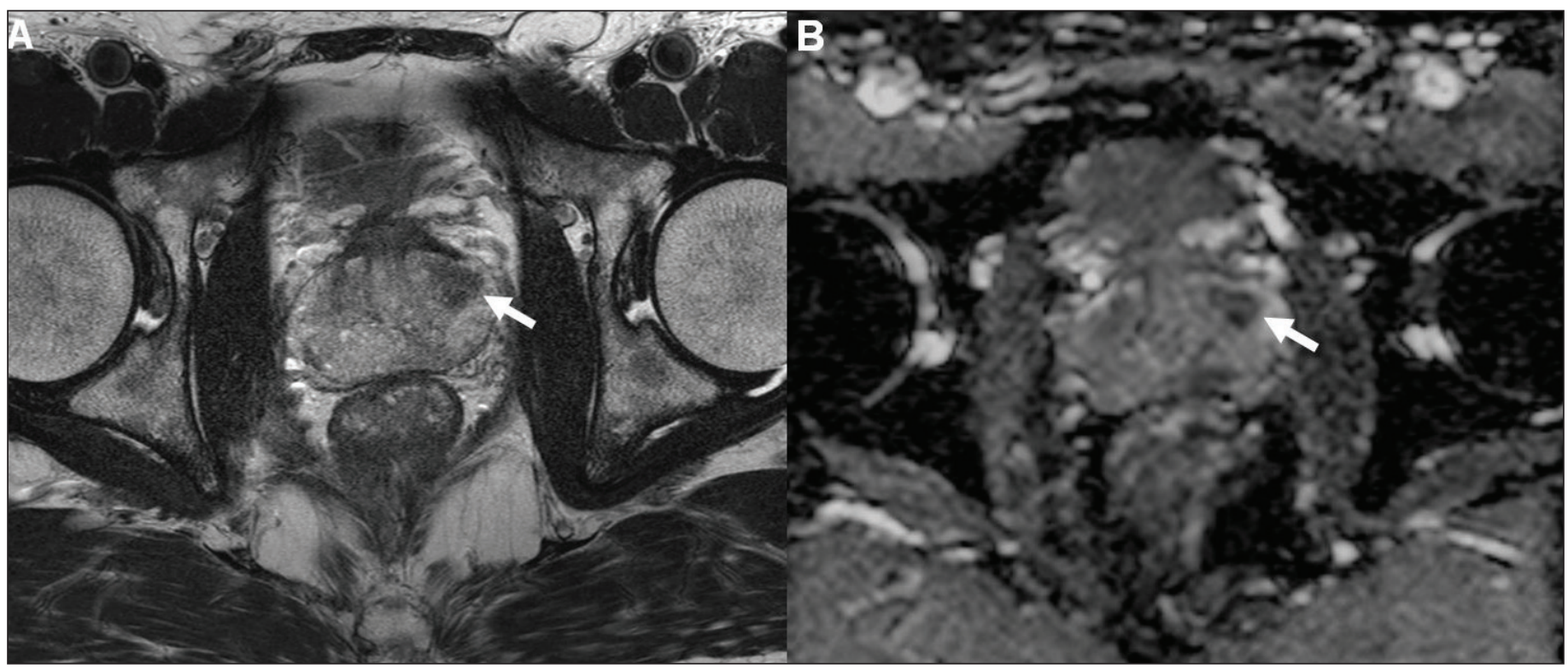

Fig. 1. A 51-year-old man with a Gleason score $3+3$ prostate cancer and a prostate-specific antigen concentration of $2.7 \mathrm{ng} / \mathrm{ml}$. A 12 -core transrectal ultrasoundguided prostate biopsy revealed Gleason $3+3$ tumour in one core in the left prostate. A radical prostatectomy specimen showed left peripheral zone cancer with Gleason $6(3+3)$ without extracapsular extension, and a tumour volume of $1.88 \mathrm{~cm}^{3}$. (A)Transverse T2-weighted image shows a low-signal lesion (arrow) in the left peripheral zone of the mid portion of the gland, suggesting possibility of cancer foci; and (B) the corresponding apparent diffusion coefficient map shows a lesion (arrow) of restricted diffusion. The apparent diffusion coefficient value of cancer is $0.880 \times 10^{-3} \mathrm{~mm}^{2} / \mathrm{sec}$. 
Kim et al.

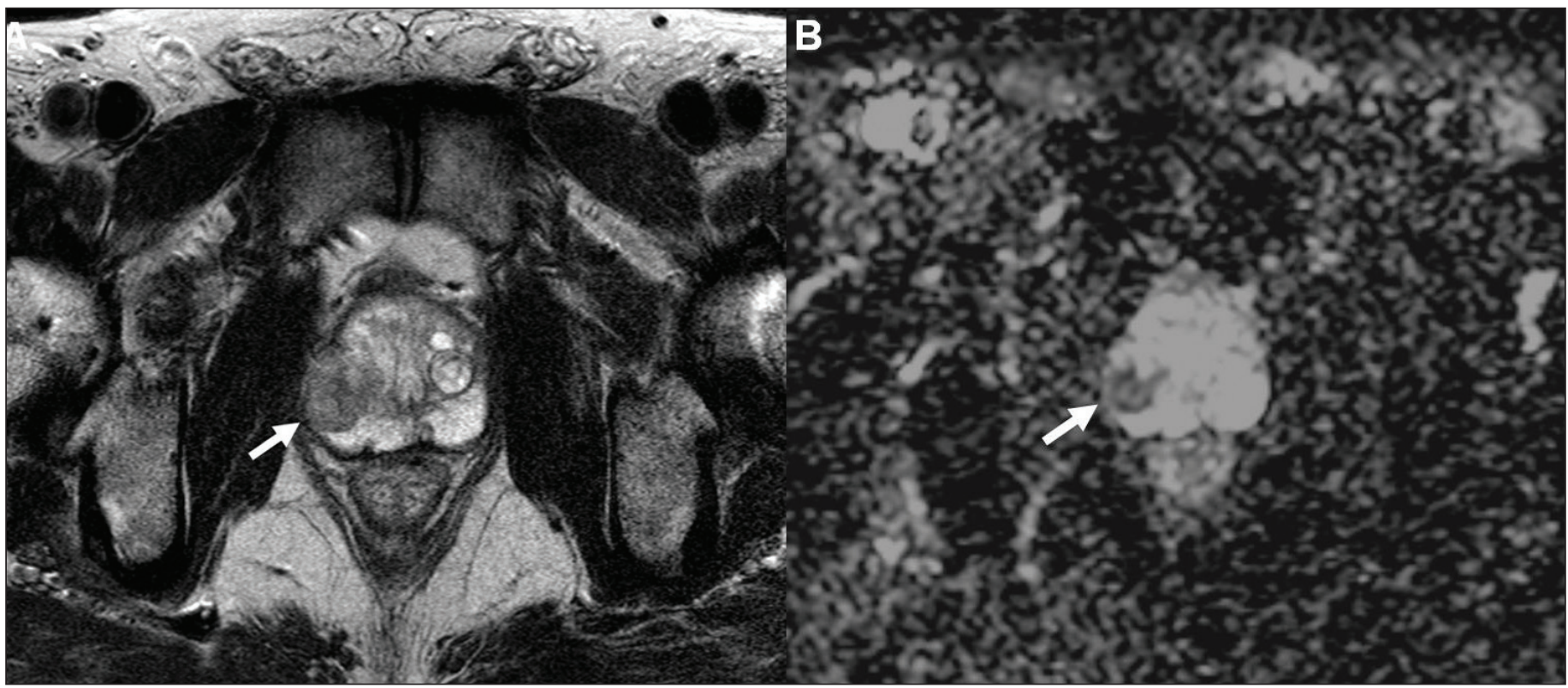

Fig. 2. A 65-year-old man with a Gleason score $4+4$ prostate cancer and a prostate-specific antigen concentration of $7.7 \mathrm{ng} / \mathrm{ml}$. A 12 -core transrectal ultrasoundguided prostate biopsy revealed Gleason $4+4$ tumour in two core in the right prostate. A radical prostatectomy specimen showed right peripheral zone cancer with Gleason $8(4+4)$ with extracapsular extension, and a tumour volume of $0.71 \mathrm{~cm}^{3}$. $(\boldsymbol{A})$ Transverse T2-weighted image shows a low-signal lesion (arrow) in the right peripheral zone of the mid portion of the gland, suggesting possibility of cancer foci; and (B) the corresponding apparent diffusion coefficient map shows a lesion (arrow) of restricted diffusion. The apparent diffusion coefficient value of cancer is $0.575 \times 10^{-3} \mathrm{~mm}^{2} / \mathrm{sec}$.

pathological GS $(3+3,3+4$, and $4+3)$. In their study, the ADC values in patients with a high GS $(4+3)$ were lower compared to those with a low GS $(3+3$ and $3+4)$. Doo et al also reported that ADC values enable the most accurate differentiation of lesions with a GS of $6\left(0.875 \times 10^{-3} \mathrm{~mm}^{2} / \mathrm{s}\right)$ from those with a GS of at least $7\left(0.779 \times 10^{-3} \mathrm{~mm}^{2} / \mathrm{s}\right) .{ }^{23}$ The

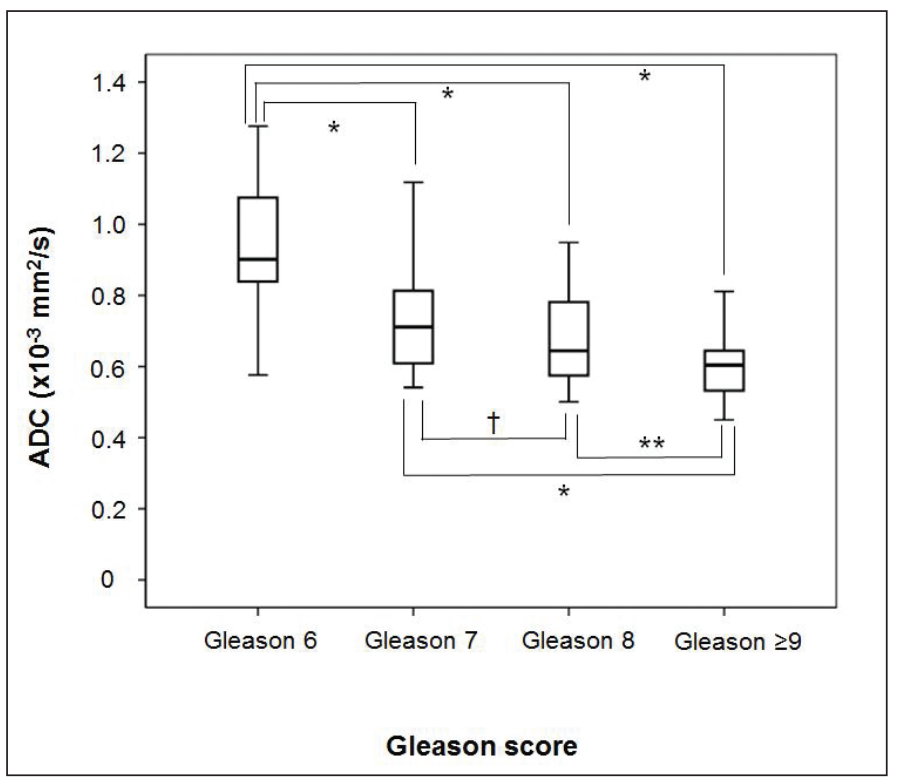

Fig.3. Box-Whisker plots of apparent diffusion coefficient (ADC) values according to Gleason score. The centre horizontal line indicates the median. ${ }^{*} p<0.001 ;{ }^{* *} p<0.01 ; \dagger p>0.05(p=0.139)$. decrease in ADC value in high GS disease can be explained by the high level of tumour cellularity, which is representative of tumour aggressiveness. Indeed, tumour cellularity is one of the major determinants for tumour grade of prostate cancer and an inverse relationship between tumour cellularity and ADC value was reported in a previous study. ${ }^{27}$

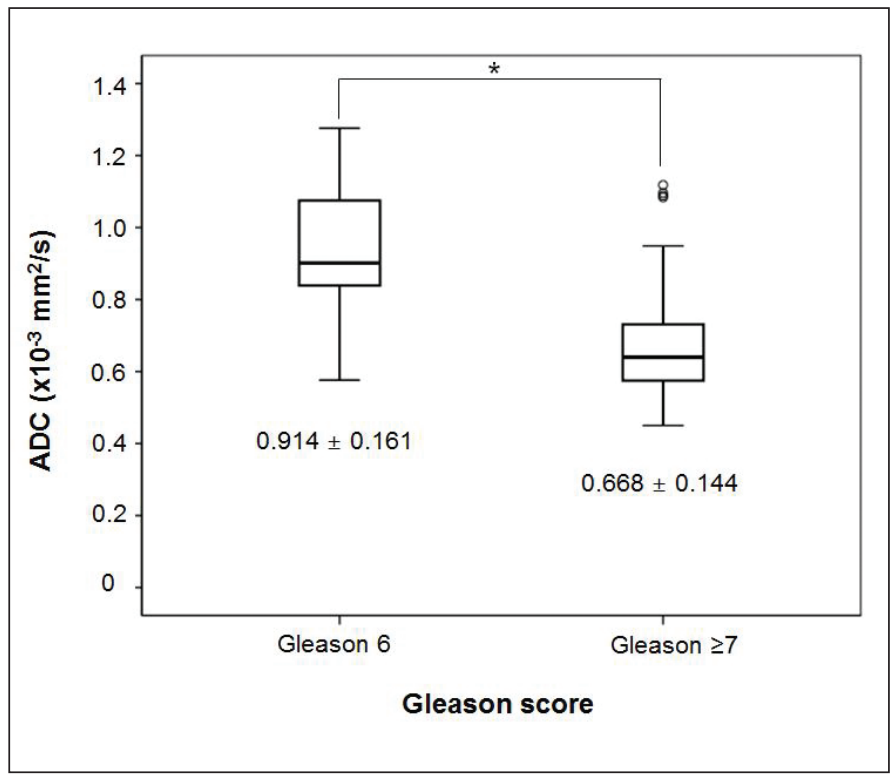

Fig. 4. Box-Whisker plots of apparent diffusion coefficient (ADC) values of Gleason score 6 disease and Gleason score $\geq 7$ disease. The centre horizontal line indicates the median. Circles denote outliers. ${ }^{*} p<0.001$. 


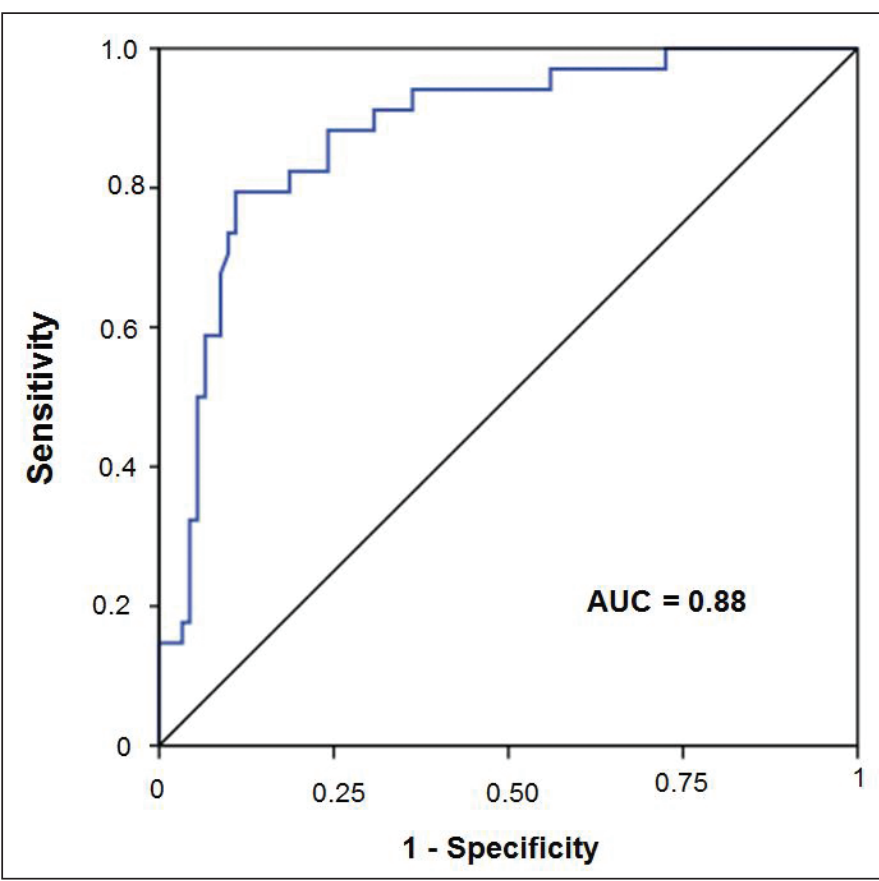

Fig. 5. Receiver operating characteristic (ROC) curve for assessment of cutoff apparent diffusion coefficient (ADC) value to predict Gleason 6 disease. Area under the curve $=0.88$.

Hambrock et $\mathrm{al}^{16}$ evaluated prostate cancer aggressiveness using a 3.0-T MRI with DWI, reporting that ADC values derived from DWI have a high discriminatory performance in the differentiation of low-, intermediate-, and high-grade prostate cancer. Consistently, we noted that higher ADC values $\left(0.830 \times 10^{-3} \mathrm{~mm}^{2} / \mathrm{s}\right)$ were significantly associated with low-risk prostate cancer (GS 6 disease), suggesting that individuals with a prostate cancer lesion having an ADC value $>0.830 \times 10^{-3} \mathrm{~mm}^{2} / \mathrm{sec}$ on $\mathrm{mpMRI}$ are more likely to have GS 6 disease compared to those who have a prostate cancer lesion with an ADC value $<0.830 \times 10^{-3} \mathrm{~mm}^{2} / \mathrm{sec}$. This finding may be helpful in the decision-making process for selecting appropriate treatment options, because patients with GS $\geq 7$ disease have a higher likelihood of greater disease extent and biochemical recurrence compared to those with GS $\leq 6$ disease. ${ }^{28}$

It is important to ensure that ADC values are measured by experienced urologists in daily clinical practice in order to help patients receive proper counselling regarding risk assessment and treatment selection. At our institution, several urologists were trained by an experienced genitourinary radiologist to recognize prostate lesions on MRI and measure ADC values. In addition, in this study, suspected tumour lesions on T2-weighted imaging and ADC maps were established through a systematic correlative review of both histopathology and MRI reports by a single urologist based on factors such as tumour location, site, and size. An experienced genitourinary radiologist (C.K.K., as an inde- pendent investigator) subsequently confirmed the regions by referencing a histopathological tumour map.

There were some limitations to the present study. First, the retrospective design of this study should be taken into account, as it may have resulted in both inherent and selection biases. Secondly, there were some limitations of this study with respect to the metrics for correlating cancerous lesions between pathological report and mpMRI. Specifically, some cancerous lesions that were not clearly visible on mpMRI were included. In patients without clearly visible tumours on ADC maps, the ROls were assessed in the area where prostate cancers were identified on pathological reports, which, despite our best efforts, may have resulted in inadvertent deviations. Third, absolute ADC values may vary between centres owing to several factors, such as the specific type of MRI scanner, imaging sequence, and b-values.

\section{Conclusion}

Our findings suggest that GS can be predicted by ADC values for tumour lesions on DWI. Furthermore, a cutoff ADC value may be useful for distinguishing GS 6 prostate cancer from prostate cancer with a GS of at least 7.

Competing interests: The authors report no competing personal or financial interests.

This paper has been peer-reviewed.

\section{References}

1. Ferlay J, Steliarova-Foucher E, Lortet-Tieulent J, et al. Cancer incidence and mortality patterns in Europe: Estimates for 40 countries in 2012. Eur J Cancer 2013;49:1374-403. http://dx.doi.org/10.1016/i. eісc.2012.12.027

2. Carozzi F, Tamburino L, Bisanzi S, et al. Are biomarkers evaluated in biopsy specimens predictive of prostate cancer aggressiveness? J Cancer Res Clin Oncol 2016;142:201-12.

3. van den Bergh $\mathrm{RC}$, Ahmed $\mathrm{HU}$, Bangma $\mathrm{CH}$, et al. Novel tools to improve patient selection and monitoring on active surveillance for low-risk prostate cancer: A systematic review. Eur Urol 2014;65:1023-31. http://dx.doi.org/10.1016/i.eururo.2014.01.027

4. Bul M, Zhu X, Rannikko A, et al. Radical prostatectomy for low-risk prostate cancer following initial active surveillance: Results from a prospective observational study. Eur Urol 2012;62:195-200. http://dx.doi. org/10.1016/i.eururo.2012.02.002

5. Okihara K, Ochiai A, Kamoi K, et al. Comprehensive assessment for novel prostate cancer markers in the prostate-specific antigen era: Focusing on Asians and Asian countries. Int J Urol 2015;22:334-41. http://dx.doi.org/10.1111/ïu.12701

6. D'Amico AV, Whittington R, Malkowicz SB, et al. Biochemical outcome after radical prostatectomy, external beam radiation therapy, or interstitial radiation therapy for clinically localized prostate cancer. JAMA 1998;280:969-74. http://dx.doi.org/10.1001/jama.280.11.969

7. Epstein J. An update of the Gleason grading system. J Urol 2010;183:433-40. http://dx.doi. org/10.1016/i.juro.2009.10.046

8. Epstein Jl, Feng Z, Trock BJ, et al. Upgrading and downgrading of prostate cancer from biopsy to radical prostatectomy: incidence and predictive factors using the modified Gleason grading system and factoring in tertiary grades. Eur Urol 2012;61:1019-24. http://dx.doi.org/10.1016/i.eururo.2012.01.050

9. Kam SC, Choi SM, Yoon S, et al. Complications of transrectal ultrasound-guided prostate biopsy: Impact of pre-biopsy enema. Korean J Urol 2014;55:732-6. htrp://dx.doi.org/10.4111/kju.2014.55.11.732

10. Fumado L, Cecchini L, Juanpere N, et al. Twelve core template prostate biopsy is an unreliable tool to select patients eligible for focal therapy. Urol Int 2015;95:197-202. http://dx.doi.org/10.1159/000381559 
Kim et al.

11. Motamedinia P, RiChard JL, McKiernan JM, et al. Role of immediate confirmatory prostate biopsy to ensure accurate eligibility for active surveillance. Urology 2012;80:1070-4. http://dx.doi.org/10.1016/i urology.2012.07.049

12. Shapiro RH, Johnstone PA. Risk of Gleason grade inaccuracies in prostate cancer patients eligible for active surveillance. Urology 2012;80:661-6. http://dx.doi.org/10.1016/j.urology.2012.06.022

13. Thompson J, Lawrentschuk $N$, Frydenberg $M$, et al. The role of magnetic resonance imaging in the diagnosis and management of prostate cancer. BJU Int 2013;112 Suppl 2:6-20. http://dx.doi.org/10.1111/ bju. 12381

14. Heidenreich A, Bellmunt J, Bolla M, et al. EAU guidelines on prostate cancer. Part I: Screening, diagnosis, and treatment of clinically localized disease. Eur Urol 2011;59:61-71. http://dx.doi.org/10.1016/i. eururo.2010.10.039

15. Junker D, Schafer G, Heidegger I, et al. Multiparametric magnetic resonance imaging/transrectal ultrasound fusion targeted biopsy of the prostate: Preliminary results of a prospective single-centre study. Urol Int 2015;94:313-8. http://dx.doi.org/10.1159/000365489

16. Hambrock T, Somford DM, Huisman HJ, et al. Relationship between apparent diffusion coefficients at 3.0-T MR imaging and Gleason grade in peripheral zone prostate cancer. Radiology 2011;259:453-61. http://dx.doi.org/10.1148/radiol.11091409

17. Nagarajan R, Margolis D, Raman S, et al. Correlation of Gleason scores with diffusion-weighted imaging findings of prostate cancer. Adv Urol 2012;2012:374805. http://dx.doi.org/10.1155/2012/374805

18. Vargas HA, Akin O, Franiel T, et al. Diffusion-weighted endorectal MR imaging at 3-T for prostate cancer: Tumour detection and assessment of aggressiveness. Radiology 2011;259:775-84. http://dx.doi. org/10.1148/radiol.11102066

19. Turkbey B, Shah VP, Pang Y, et al. Is apparent diffusion coefficient associated with clinical risk scores for prostate cancers that are visible on 3-T MR images? Radiology 2011;258:488-95. http://dx.doi. org/10.1148/radiol.10100667

20. Kim TH, Jeong JY, Lee SW, et al. Diffusion-weighted magnetic resonance imaging for prediction of insignificant prostate cancer in potential candidates for active surveillance. Eur Radiol 2015;25:1786-92. http://dx.doi.org/10.1007/s00330-014-3566-2
21. Park SY, Kim CK, Park BK, et al. Prediction of biochemical recurrence following radical prostatectomy in men with prostate cancer by diffusion-weighted magnetic resonance imaging: Initial results. Eur Radiol 2011;21:1111-8. http://dx.doi.org/10.1007/s00330-010-1999-9

22. Noguchi M, Stamey TA, McNeal JE, et al. Assessment of morphometric measurements of prostate carcinoma volume. Cancer 2000;89:1056-64. http://dx.doi.org/10.1002/10970142(20000901)89:5<1056::AID-CNCR15>3.0.C0;2-U

23. Doo KW, Sung DJ, Park BJ, et al. Detectability of low- and intermediate- or high-risk prostate cancer with combined T2-weighted and diffusion-weighted MRI. Eur Radiol 2012;22:1812-9. http://dx.doi. org/10.1007/s00330-012-2430-5

24. Shigemura K, Yamanaka N, Yamashita M. Can diffusion-weighted magnetic resonance imaging predict a high Gleason score of prostate cancer? Korean J Urol 2013;54:234-8. http://dx.doi.org/10.4111/ kju.2013.54.4.234

25. Caivano R, Rabasco P, Lotumolo A, et al. Comparison between Gleason score and apparent diffusion coefficient obtained from diffusion-weighted imaging of prostate cancer patients. Cancer Invest 2013;31:625-9. http://dx.doi.org/10.3109/07357907.2013.845673

26. Hambrock T, Hoeks C, Hulsbergen-van de Kaa C, et al. Prospective assessment of prostate cancer aggressiveness using 3-T diffusion-weighted magnetic resonance imaging-guided biopsies vs. a systematic 10-core transrectal ultrasound prostate biopsy cohort. Eur Urol 2012;61:177-84. http://dx.doi.org/10.1016/i. eururo.2011.08.042

27. Donati OF, Mazaheri Y, Afaq A, et al. Prostate cancer aggressiveness: Assessment with whole-lesion histogram analysis of the apparent diffusion coefficient. Radiology 2014;271:143-52. http://dx.doi. org/10.1148/radiol.13130973

28. Carter HB, Partin AW, Walsh PC, et al. Gleason score 6 adenocarcinoma: Should it be labeled as cancer? J Clin Oncol 2012;30:4294-6. http://dx.doi.org/10.1200/JC0.2012.44.0586

Correspondence: Dr. Seong Soo Jeon, Department of Urology, Samsung Medical Centre, Sungkyunkwan University School of Medicine, Seoul, Korea; seongsoo.jeon@samsung.com 